\title{
Disrupted relationship between "resting state" connectivity and task-evoked activity during social perception in schizophrenia
}

\author{
Sjoerd J.H. Ebisch a,b,*, Vittorio Gallese ${ }^{c, d}$, Anatolia Salone ${ }^{a}$, Giovanni Martinotti ${ }^{a, i}$, Giuseppe di Iorio a, \\ Dante Mantini ${ }^{\mathrm{e}, \mathrm{f}, \mathrm{g}}$, Mauro Gianni Perrucci ${ }^{\mathrm{a}, \mathrm{b}}$, Gian Luca Romani ${ }^{\mathrm{a}, \mathrm{b}}$, \\ Massimo Di Giannantonio ${ }^{\mathrm{a}}$, Georg Northoff ${ }^{\mathrm{h}, \mathrm{j}}$ \\ a Department of Neuroscience, Imaging and Clinical Sciences, G. d'Annunzio University of Chieti-Pescara, Via dei Vestini 31, 66013 Chieti, Italy \\ ${ }^{\mathrm{b}}$ Institute of Advanced Biomedical Technologies (ITAB), G. d'Annunzio University of Chieti-Pescara, Via dei Vestini 31, 66013 Chieti, Italy \\ c Department of Medicine and Surgery, Section of Neuroscience, University of Parma, Via Volturno 39E, 43125 Parma, Italy \\ d Institute of Philosophy, School of Advanced Study, University of London, London, UK \\ e Department of Health Sciences and Technology, ETH Zurich, Zurich, Switzerland \\ ${ }^{\mathrm{f}}$ Department of Experimental Psychology, University of Oxford, Oxford, UK \\ ${ }^{g}$ Research Center for Motor Control and Neuroplasticity, KU Leuven, Tervuursevest 101, 3001 Leuven, Belgium

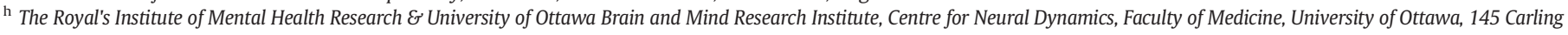 \\ Avenue, Rm. 6435, Ottawa, ON K1Z 7K4, Canada \\ i University of Hertfordshire, Department of Pharmacy, Pharmacology, Clinical Sciences, Herts, UK \\ ${ }^{j}$ Mental Health Centre, Zhejiang University School of Medicine, Hangzhou, Zhejiang Province, China
}

\section{A R T I C L E I N F O}

\section{Article history:}

Received 9 November 2016

Received in revised form 5 July 2017

Accepted 7 July 2017

Available online $\mathrm{xxxx}$

\section{Keywords:}

Psychosis

Functional magnetic resonance imaging

Intrinsic functional connectivity

Social cognition

Stimulus congruency

\begin{abstract}
A B S T R A C T
Schizophrenia has been described as a self-disorder, whereas social deficits are key features of the illness. Changes in "resting state" activity of brain networks involved in self-related processing have been consistently reported in schizophrenia, but their meaning for social perception deficits remains poorly understood. Here, we applied a novel approach investigating the relationship between task-evoked neural activity during social perception and functional organization of self-related brain networks during a "resting state".

"Resting state" functional MRI was combined with task-related functional MRI using a social perception experiment. Twenty-one healthy control participants ( $\mathrm{HC}$ ) and 21 out-patients with a diagnosis of schizophrenia $(\mathrm{SCH})$ were included. There were no significant differences concerning age, IQ education and gender between the groups. Results showed reduced "resting state" functional connectivity between ventromedial prefrontal cortex and dorsal posterior cingulate cortex in SCH, compared to HC. During social perception, neural activity in dorsal posterior cingulate cortex and behavioral data indicated impaired congruence coding of social stimuli in SCH. Task-evoked activity during social perception in dorsal posterior cingulate cortex co-varied with dorsal posterior cingulate cortex-ventromedial prefrontal cortex functional connectivity during a "resting state" in HC, but not in SCH. Task-evoked activity also correlated with negative symptoms in SCH.

These preliminary findings, showing disrupted prediction of social perception measures by "resting state" functioning of self-related brain networks in schizophrenia, provide important insight in the hypothesized link between self and social deficits. They also shed light on the meaning of "resting state" changes for tasks such as social perception.
\end{abstract}

(C) 2017 Elsevier B.V. All rights reserved.

\section{Introduction}

Schizophrenia has been described as being a self-disorder that is expressed in a heterogeneity of symptoms (Northoff, 2014; Nelson, Fornito, et al., 2009; Sass and Parnas, 2003). Among these symptoms,

\footnotetext{
* Corresponding author at: Department of Neuroscience, Imaging and Clinical Sciences ITAB, G. d'Annunzio University of Chieti-Pescara, Via Dei Vestini 31, 66013 Chieti (CH), Italy.

E-mail address: s.ebisch@unich.it (S.J.H. Ebisch).
}

social impairments have been recognized as key features of the illness (Green et al., 2012; Horan et al., 2012; Fett et al., 2011; Eack et al., 2010; Addington et al., 2008; Couture et al., 2006; Minkowski, 1927). However, the exact relation between social impairments and self-disorder as a disturbed sense of self remains unclear.

Self-related processing regards a well-studied aspect constituting our sense of self. It concerns the neural processing of the relation of environmental/exteroceptive and bodily/interoceptive stimuli with one's own person (van der Meer et al., 2010; Northoff 2016). If that is disrupted, such stimuli cannot be properly related to the self. 
Because environmental stimuli include social stimuli concerning other individuals' self, it raises the question how self-related processing in schizophrenia is linked to social deficits (Haug et al., 2014; Nelson, Sass, et al., 2009; Gallese, 2003; Parnas et al., 2002).

Studies in healthy participants suggest that social perception of others' mental and bodily states could be mediated by self-related processing (Schilbach et al., 2008; Fisher et al., 2008; Mitchell et al., 2005). The integration of multiple signals during social perception has recently been associated with self-related processing: congruent social information could increase self-relatedness and facilitate subjective judgments of others' experiences (Ebisch et al., 2016). This is in line with accounts proposing association of self-relatedness with other functions (Northoff, 2016), including integrative information processing (Sui and Humphreys, 2015).

The present study aimed at investigating whether deficits in the integration of multiple signals during social perception can be related to disrupted functional organization of self-related brain networks in schizophrenia.

Self-related processing involves cortical midline structures (CMS), like anterior (ACC) and posterior cingulate cortex (PCC), and medial prefrontal cortex (MPFC) (Qin and Northoff, 2011; van der Meer et al., 2010). These CMS are also known to be involved in social cognition (Schilbach et al., 2008; Mitchell et al., 2005) interacting with sensorimotor and affective systems for social understanding (Molnar-Szakacs and Uddin, 2013; Gallese, 2007). Furthermore, self-related activity within CMS shows strong overlap with spontaneous activity during task-unrelated thought (i.e., "resting state") in the default mode network (DMN) (Qin and Northoff, 2011; Whitfield-Gabrieli et al., 2011; Bai et al., 2016; Huang et al., 2016).

"Resting state" changes in MPFC and PCC have indeed been shown in schizophrenia (Sheffield and Barch, 2016; Northoff, 2015; Kuhn and Gallinat, 2013; van der Meer et al., 2010). These changes included functional connectivity within CMS as well as with sensorimotor and affective networks (Martino et al., 2017; Berman et al., 2016). However, the meaning of such "resting state" changes for subsequent task-evoked activity during social perception remains poorly understood. Specifically, it is unclear whether the relationship between CMS "resting state" functional connectivity (RS-FC) and task-evoked impairments in social perception is altered in schizophrenia.

For this purpose, we combined "resting state" RS-FC functional magnetic resonance imaging (fMRI) with task-related fMRI using a social perception experiment in schizophrenia.

RS-FC analysis provides a basic index of functional network structure in terms of long-range communication between brain regions based on low-frequency fluctuations of the Blood Oxygen Level Dependent (BOLD) signal. We focused on RS-FC of the regions of interest (ROIs) ventral MPFC and PCC showing overlap with self-related processing and high "resting state" activity according to meta-analyses (Qin and Northoff, 2011). The social perception experiment addressed integrative processing of visual social stimuli allowing to compare the processing of congruent and incongruent information from multiple sources (others' sensorimotor experiences and facial expressions of emotion).

Analyses preliminarily tested the hypotheses that patients with schizophrenia, compared to healthy control participants, are characterized by (i) decreased RS-FC of vMPFC and PCC with CMS, sensorimotor or affective networks; (ii) impaired performance and task-evoked activity in nodes of self-networks during a social perception task requiring the integrative processing of congruent versus incongruent social stimulus content; (iii) a decreased relation, that is correlation, between RS-FC and task-evoked activity during social perception including their relation to psychopathological symptoms.

\section{Material and methods}

\subsection{Participants}

Twenty-one adult, healthy, right-handed control participants (HC) and twenty-one adult, right-handed out-patients with a diagnosis of schizophrenia ( $\mathrm{SCH}$ ) according to DSM-IV criteria were included in the present study. Detailed evaluation procedures are described in the Supplementary material. Demographic and clinical data are provided in Table 1. $t$-Tests did not show significant differences between the HC and $\mathrm{SCH}$ groups for age, education (years) and intelligence quotient (IQ) (see Table 1). A Chi-square test showed no significant difference in gender distribution between the groups.

All participating patients were rated for symptom severity with the Positive and Negative Symptom Scale (Kay et al., 1987). Exclusion criteria for all participants included physical health problems and neurological hard signs, standard contraindications for fMRI, a history of severe head trauma, loss of consciousness, drug abuse, IQ $<70$, lifetime intake of neuroleptic/typical antipsychotic drugs, and, specifically for the HC group, a personal history of Axis I/II disorders or a history of psychosis in first-degree relatives.

The experimental protocol was approved by the local institutional ethics committee. Written informed consent was obtained from all participants prior to participation, in line with the Declaration of Helsinki.

\section{2. fMRI procedures}

Each participant underwent BOLD contrast fMRI scanning at $3 \mathrm{~T}$ and completed two task-free fMRI scanning runs ("resting state" 2 $\times 5.2 \mathrm{~min})$ and four "task-related" fMRI scanning runs ( $4 \times 7.7 \mathrm{~min})$. Scanning details and fMRI preprocessing procedures are described in the Supplementary material.

To control for effects due to motion inside the scanner, FMRI preprocessing included regression of fMRI motion parameters and scrubbing of motion affected functional volumes (Power et al., 2012). FMRI runs with movements exceeding $3 \mathrm{~mm}$ were excluded from analysis. Finally, the $\mathrm{HC}$ and SCH groups were compared for motion inside the scanner: no significant differences were found (Table 1 ).

Table 1

Participant characteristics.

\begin{tabular}{|c|c|c|}
\hline & $\begin{array}{l}\text { Healthy } \\
\text { control group }\end{array}$ & $\begin{array}{l}\text { Schizophrenia } \\
\text { group }\end{array}$ \\
\hline $\begin{array}{l}\text { Gender }^{\mathrm{a}} \\
\text { (male/female) }\end{array}$ & $16 / 5$ & $16 / 5$ \\
\hline $\begin{array}{l}\text { Age }^{\mathrm{b}} \\
\text { (mean years, standard deviation) }\end{array}$ & $30.84 \pm 4.59$ & $32.24 \pm 7.33$ \\
\hline $\begin{array}{l}\text { Intelligence quotient }{ }^{\mathrm{c}} \\
\text { (mean IQ, standard deviation) }\end{array}$ & $117 \pm 13$ & $111 \pm 14$ \\
\hline $\begin{array}{l}\text { Education level (mean years of education, } \\
\text { standard deviation })^{\mathrm{d}}\end{array}$ & $13.19 \pm 2.25$ & $12.95 \pm 2.67$ \\
\hline $\begin{array}{l}\text { Illness duration } \\
\text { (mean years, standard deviation) }\end{array}$ & n.a. & $5.19 \pm 2.36$ \\
\hline $\begin{array}{l}\text { Psychotic episodes } \\
\text { (mean number, standard deviation) }\end{array}$ & n.a. & $1.76 \pm 1.45$ \\
\hline $\begin{array}{l}\text { Chlorpromazine equivalences } \\
\text { (mean CZP, standard deviation) }\end{array}$ & n.a. & $265.25 \pm 136.63$ \\
\hline $\begin{array}{l}\text { PANSS positive symptoms } \\
\text { (mean score, standard deviation) }\end{array}$ & n.a. & $15.57 \pm 3.71$ \\
\hline $\begin{array}{l}\text { PANSS negative symptoms } \\
\text { (mean score, standard deviation) }\end{array}$ & n.a. & $19.43 \pm 3.78$ \\
\hline 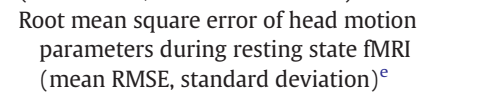 & $0.21 \pm 0.16$ & $0.21 \pm 0.1$ \\
\hline $\begin{array}{l}\text { Root mean square error of head motion } \\
\text { parameters during task fMRI (mean RMSE, } \\
\text { standard deviation) }\end{array}$ & $0.22 \pm 0.15$ & $0.26 \pm 0.21$ \\
\hline 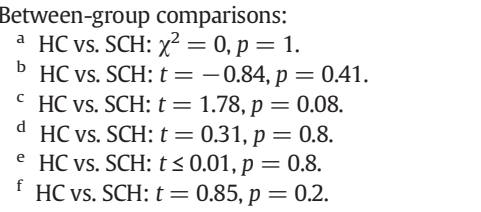 & & \\
\hline
\end{tabular}


During task-free fMRI scanning, all participants were instructed to watch a white fixation cross at the centre of a black screen and to think freely.

Regarding task-related fMRI, participants were involved in a "social perception" task (see Supplementary material and Ebisch et al., 2016). Briefly, according to a rapid event-related fMRI paradigm, a series of video clips ( $2400 \mathrm{~ms}$ ) was randomly shown to the participant depicting an individual being hit or caressed by another individual, while facially expressing pain or pleasure. Videos could be categorized in four experimental conditions based on the combination of "tactile sensation" and "facial expression" which could be either congruent or incongruent: 1) caress-pleasure, 2) hit-pain, 3) caress-pain, and 4) hit-pleasure.

Twenty five percent of the videos were randomly followed by the question: "Please indicate by means of a button press whether you find that the emotional expression (pleasure, pain) in the last video was correct or incorrect with respect to the tactile sensation (caress, hit)".

\subsection{Analysis: task-free fMRI}

To investigate RS-FC patterns, a seed-based, whole-brain, voxel-wise analysis was performed on the fMRI time series obtained during taskfree fMRI scanning (see Supplementary material) (Ebisch et al., 2011; Van Dijk et al., 2010; Fox and Raichle, 2007).

RS-FC indices were calculated as correlations between BOLD fluctuations in the seed ROIs (extracted from and averaged across the voxels included in the ROI) and BOLD fluctuations in all individual brain voxels. Seed ROIs were defined as spheres (radius $=6 \mathrm{~mm}$ ) and based on coordinates specifically associated with self-related and familiarity processing

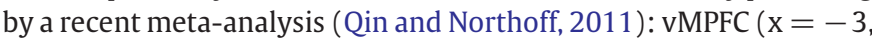
$\mathrm{y}=49, \mathrm{z}=15)$ and PCC $(\mathrm{x}=1, \mathrm{y}=-62, \mathrm{z}=19)$.

Random effect independent-sample $t$-tests were performed to compare the RS-FC maps of each group for the VMPFC and PCC seed ROIs. RSFC indices were used as dependent variable and group as independent variable. Group contrast RS-FC maps were corrected for multiple comparisons by the false discovery rate (FDR).

Two SCH participants were excluded from this analysis due to excessive head motion ( $>3 \mathrm{~mm}$ ), but there were no significant differences between the remaining $\mathrm{HC}$ and $\mathrm{SCH}$ groups for gender distribution $(p=0.84)$, education $(p=0.61), \mathrm{IQ}(p=0.15)$ and age $(p=0.28)$.

\subsection{Analysis: task fMRI}

A ROI-based random effect analysis of variance (ANOVA) was performed on the task-related fMRI data. ROIs were based on the coordinates of the seed ROIs used for the RS-FC analysis (VMPFC, PCC) and the peak coordinates of clusters showing differential RS-FC patterns in the SCH group (dPCC and mid/anterior insula). Individual BOLD responses to the experimental conditions (beta-values) were extracted from and averaged across the voxels included in the ROIs.

Group (HC, SCH) was used as between-subject variable, whereas "tactile sensation" (hit, caress) and "facial expression" (pain, pleasure) were used as within-subject variables. Statistical significance of effects related to the experimental factors were corrected for multiple comparisons by multiplying two-tailed $p$-values by the number of ROIs: vMPFC, vPCC, dPCC, left anterior insula.

Three SCH participants were excluded from this analysis due to excessive head motion ( $>3 \mathrm{~mm}$ ) or difficulties in completing the task. These were not the same patients as those excluded for the task-free fMRI analysis. Because the resulting groups were not matched for IQ, the two HC participants with the highest IQ scores were excluded. After this there were no significant differences between the groups concerning gender distribution $(p=0.41)$, education $(p=0.99)$, IQ $(p=0.06)$ and age $(p=0.57)$.

\subsection{Analysis: rest-task relationship}

We tested whether the $\mathrm{SCH}$ group was characterized by an altered relationship between task-related neural activity during the social perception task and RS-FC of self-network nodes. Both task-free data and task-related fMRI data were available from 16 SCH and 19 HC participants. No significant differences were detected between these groups for gender distribution ( $p=0.64)$, education $(p=0.84)$, IQ ( $p=$ $0.11)$ and age $(p=0.40)$.

Correlations (Spearman's rho) were calculated between (i) RS-FC of the original seed ROIs with ROIs showing altered RS-FC patterns as well as altered task-related responses in the SCH group, and (ii) differential beta-values for the congruent and the incongruent social stimuli in ROIs that showed altered RS-FC patterns as well as altered task-related

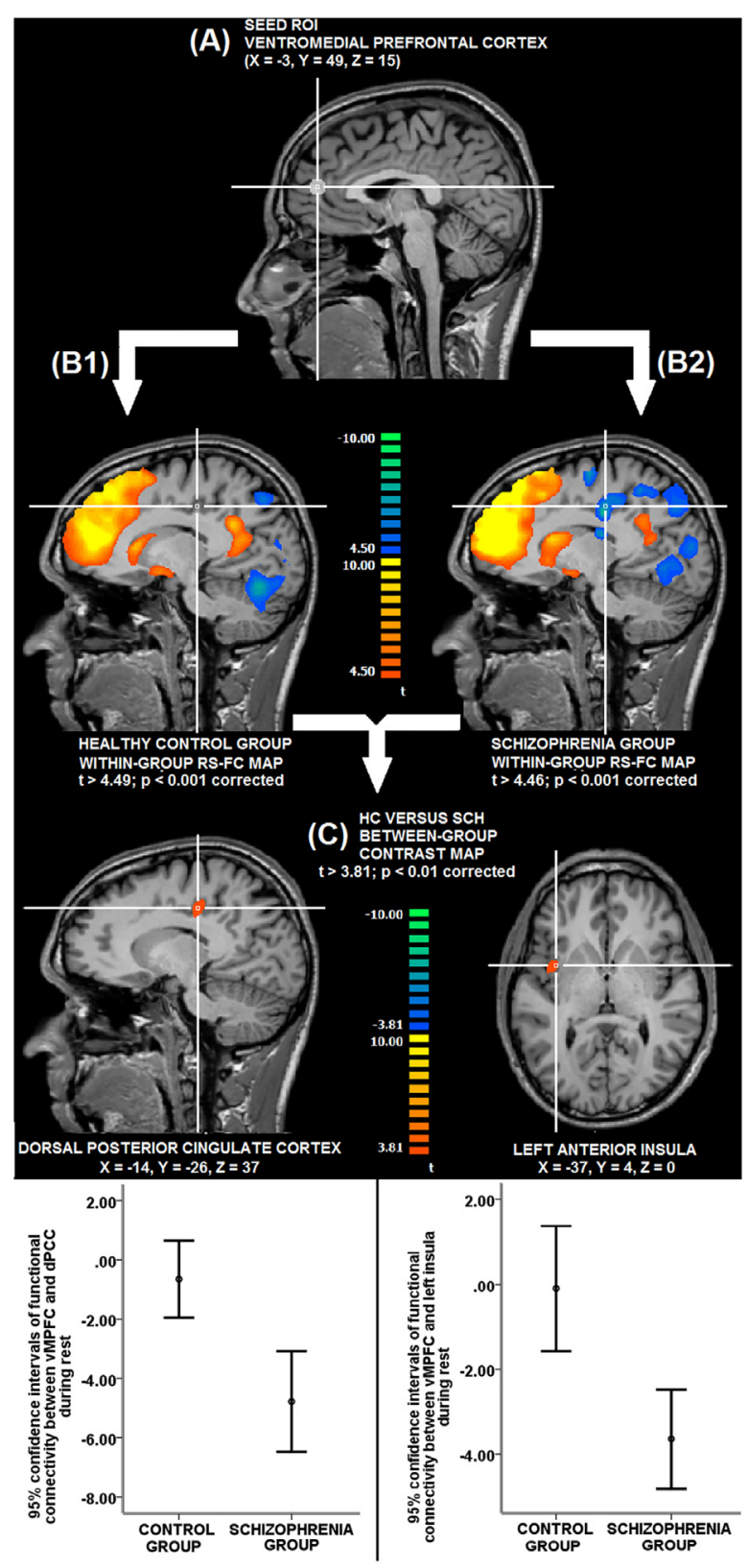

Fig. 1. Task-free fMRI results: (A) Seed ROI vMPFC; (B) group statistical RS-FC maps for the vMPFC seed ROI in the HC group (B1) and the SCH group (B2) separately; (C) group comparisons between the vMPFC RS-FC maps showing reduced RS-FC in the SCH group, compared to the HC group, in $\mathrm{dPCC}$ (left) and left anterior insula (right) with corresponding graphs representing RS-FC indices (z-scores) in the HC and SCH group (below). 
responses in the $\mathrm{SCH}$ group. Statistical significance was corrected for multiple comparisons by multiplying the obtained $p$-values by the number of correlations calculated. Correlations were bootstrapped $(10,000$ samples) for accuracy estimation.

\section{Results}

\subsection{Task-free fMRI: RS-FC of $v M P F C$ and PCC}

Voxel-wise analysis yielded weaker RS-FC of vMPFC (Fig. 1A) with voxel clusters in dorsal PCC (dPCC; Brodmann Area, BA, 5; cluster size $=729 \mathrm{~mm}^{3}$ ) and left mid/anterior insula (BA 13; cluster size = $\left.864 \mathrm{~mm}^{3}\right)$ in the SCH group, compared to the HC group $(t \geq 3.81 ; p \leq$ $0.0005, \mathrm{q} \leq 0.01$ after FDR correction) (Fig. 1B). Repeating the same analysis without applying global signal regression confirmed this result by showing similar patterns ( $t \geq 4.6 ; p \leq 0.00005, \mathrm{q} \leq 0.001$ after FDR correction) (Supplementary Fig. 1 ). Therefore, it is unlikely that group differences can be attributed to secondary effects of global signal regression (Yang et al., 2014; Fox et al., 2009). No significant RS-FC differences were found between the HC and SCH groups for the PCC seed ROI. Correlation analysis did not reveal any significant correlations between RS-FC (vMPFC-PCC or vMPFC-insula), and medication or illness duration (all $p>0.3$ ).

Graphs in Fig. 1C provide mean RS-FC indices (z-values) and 95\% confidence intervals $(\mathrm{CI})$.

\subsection{Task fMRI: differentiation between congruent and incongruent social stimuli}

ROI-based ANOVA of neural activation patterns during the social perception fMRI task showed a significant group $(\mathrm{HC}, \mathrm{SCH}) \times$ tactile sensation (hit, caress) $\times$ facial expression (pain, pleasure) interaction effect in the dPCC ROI $\left(F_{1,35}=8.127, p \leq 0.05\right.$ after Bonferroni correction for multiple comparisons) (Fig. 2A), but not in left mid/anterior insula,
vMPFC or PCC (all $p>0.1$ ). The 3-way interaction effect ("facial expression" $\times$ "tactile sensation" $\times$ "group") indicates a difference in congruence coding (i.e., integrative processing) between the $\mathrm{HC}$ and SCH groups.

Post-hoc analysis on dPCC activity in the HC group showed a significant tactile sensation (hit, caress) $\times$ facial expression (pain, pleasure) interaction effect $\left(F_{1,18}=18.514, p<0.001\right)$, but no effect of tactile sensation $(p>0.5)$ or facial expression $(p>0.3)$ (Fig. 2B upper graph). By contrast, no significant effect of experimental condition on dPCC activity was found in the $\mathrm{SCH}$ group (all $p>0.5$ ) (Fig. 2B lower graph).

In sum, the 3-way interaction effect in $\mathrm{APCC}$ was driven by stronger BOLD responses in the $\mathrm{HC}$ group for the congruent stimuli, compared to the incongruent stimuli, whereas this difference was absent in the $\mathrm{SCH}$ group (Fig. 2C).

Neither the group $(\mathrm{HC}, \mathrm{SCH}) \times$ tactile sensation (hit, caress) interaction effect nor the group $(\mathrm{HC}, \mathrm{SCH}) \times$ facial expression (pain, pleasure) interaction effect was significant in $\mathrm{dPCC}$, left mid/anterior insula, vMPFC or vPCC (all $p>0.2$ ).

Correlation analysis ruled out the impact of medication and illness duration on signal changes in dPCC during social perception (i.e., differential beta-values for the congruent and the incongruent social stimuli) in the SCH group (all $p>0.4$ ).

\subsection{Relationship between task-free and task fMRI}

Investigating the relationship between neural activity in dPCC during the social perception task (altered in $\mathrm{SCH}$, compared to $\mathrm{HC}$ ) and RS-FC of dPCC with the vMPFC seed ROI (also altered in SCH), a significant negative correlation was found in the HC group $(r=-0.64$; standard error $=0.16 ; p<0.01)$, but not in the SCH group $(r=-0.05$; standard error $=0.32 ; p>0.5$ ) (Fig. 3). Specifically, in the HC group, weaker vMPFC-dPCC RS-FC during task-free fMRI scanning predicted stronger differential neural responses in dPCC between the congruent and the incongruent stimuli during task-related neural activity. By

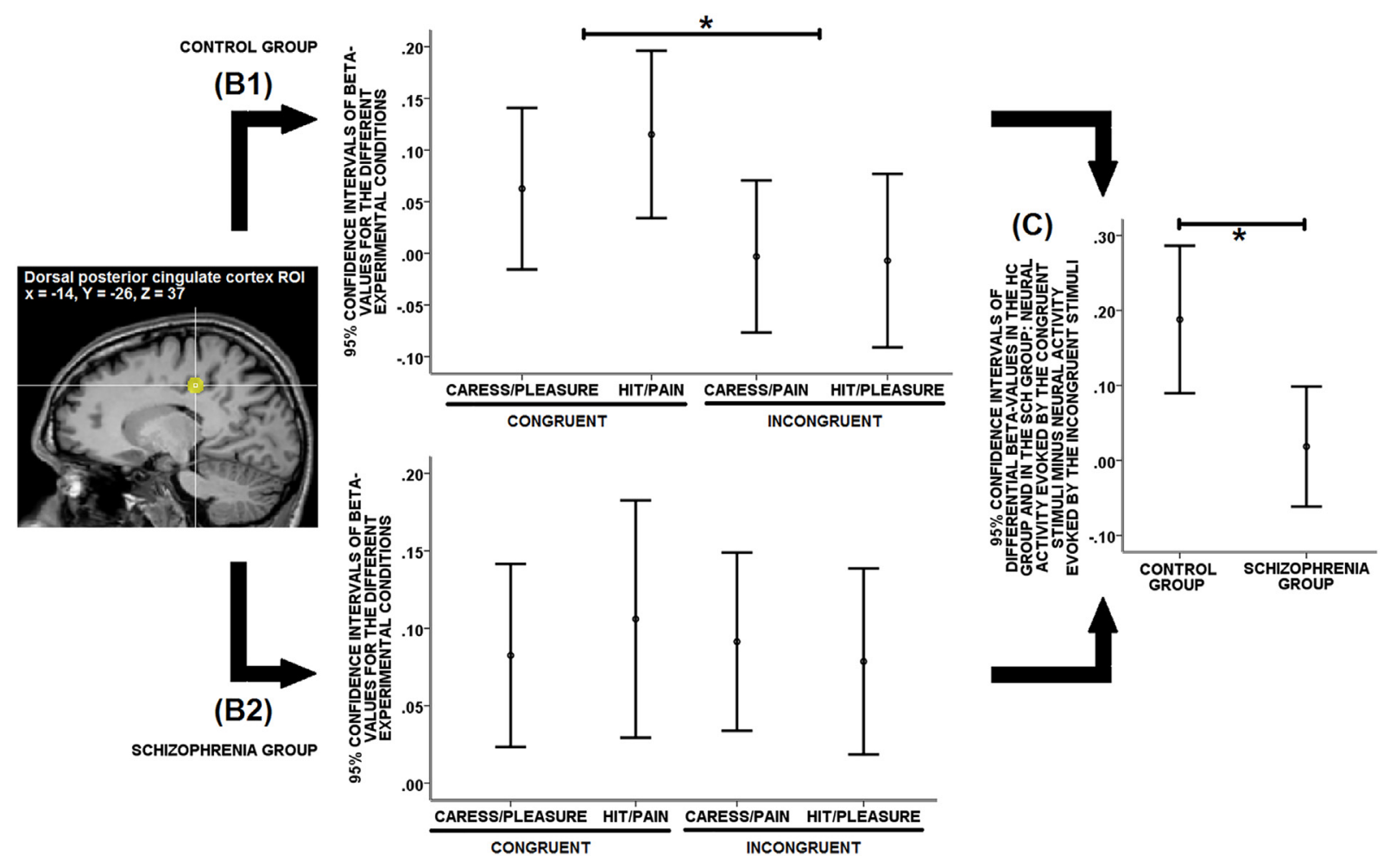

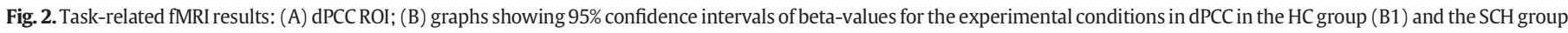

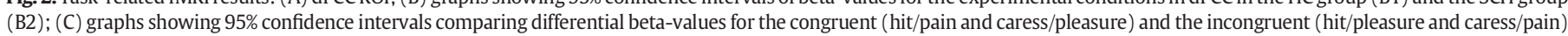
conditions in $\mathrm{APCC}$ between the HC group and the SCH group. *Statistically significant difference $p<0.001$. 

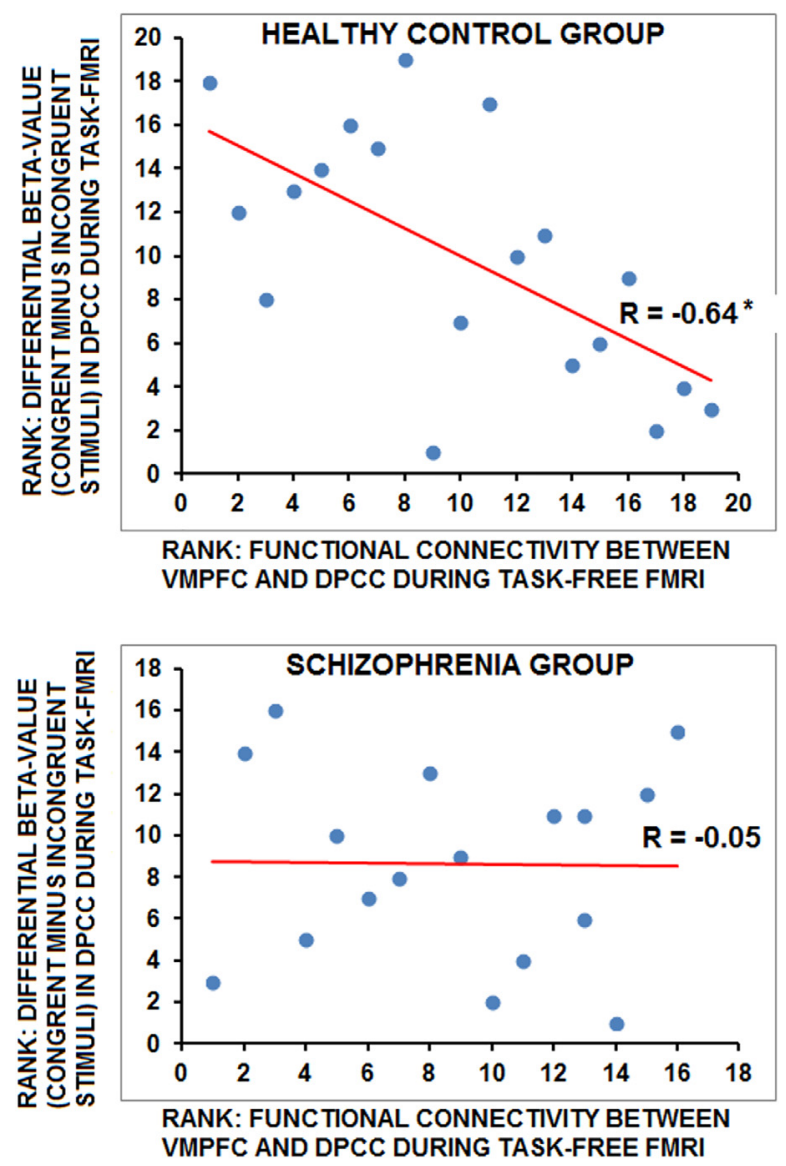

Fig. 3. Scatter plots showing the relationship between task-free fMRI data (RS-FC of vMPFC with $\mathrm{dPCC}$ ), and task-related fMRI data (differential beta-values for the contrast between congruent and incongruent stimuli in dPCC). *Statistically significant correlation $p<0.05$ corrected.

contrast, in the SCH group task-related neural activity in dPCC could not be predicted by dPCC-vMPFC RS-FC.

\subsection{Relationship between task fMRI and psychopathological symptoms}

Concerning illness severity, correlations were calculated focusing on altered task-free and task fMRI data in SCH, that is, vMPFC-dPCC RS-FC and congruence coding in $\mathrm{APCC}$, respectively. No significant correlations were found between vMPFC-dPCC RS-FC and positive or negative symptoms. A significant negative correlation was detected between differential BOLD responses in $\mathrm{APCC}$ for the congruent and incongruent stimuli, and negative symptoms $(r=-0.55$; standard error $=0.17 ; p \leq 0.05$ after correction for multiple comparisons) (Fig. 4), but not positive symptoms $(p>0.5)$.

\section{Discussion}

Summarizing, the main findings of the present fMRI study in the $\mathrm{SCH}$ group, compared to the HC group, are as follows: (i) decreased RS-FC of vMPFC with dPCC; (ii) impairment in task-evoked activity in the same dPCC during social perception with decreased discrimination between congruent and incongruent social content; (iii) lack of co-variance between dPCC task-evoked activity and vMPFC-dPCC RS-FC; (iv) correlation of altered dPCC task-evoked activity with illness severity. These findings imply that disrupted RS-FC of brain networks associated with self-related processing may contribute to social perception deficits in schizophrenia. It also sheds light on the meaning of changes in functional network properties (i.e., functional organization) during the "resting

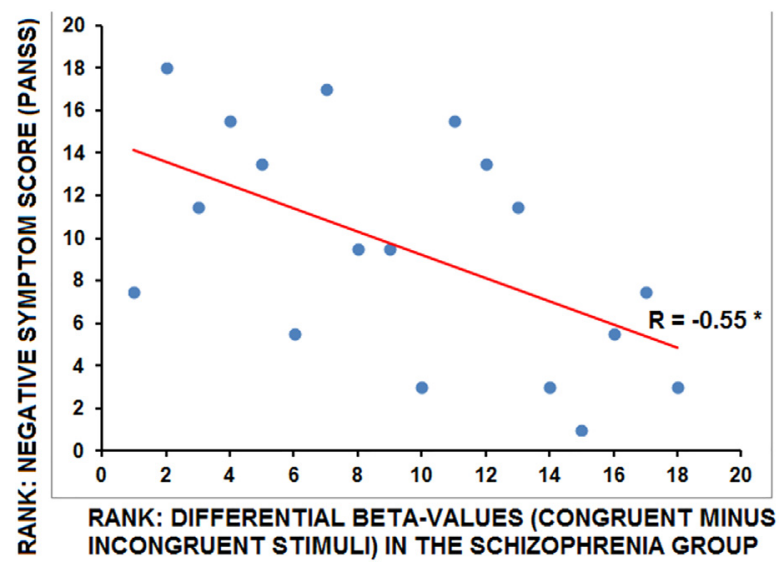

Fig. 4. Scatter plots showing the relationship between task fMRI (differential beta-values for the contrast between congruent and incongruent stimuli in dPCC) and psychopathological symptoms. *Statistically significant correlation $p<0.05$ corrected.

state" for subsequent task-evoked activity and behavior such as social perception (Weinberger and Radulescu, 2016).

Our task-free fMRI results showing decreased RS-FC of vMPFC in schizophrenia are in accordance with previous studies that reported altered RS-FC of CMS (Schilbach et al., 2016; van der Meer et al., 2010). Because the vMPFC ROI overlaps with self-related processing (Qin and Northoff, 2011), one may speculate that these RS-FC abnormalities could be closely related to abnormalities in self processing (Northoff, 2014; Nelson, Fornito, et al., 2009; Vinogradov et al., 2008; Sass and Parnas, 2003). Additionally, the dPCC cluster showing altered RS-FC with vMPFC is localized in the medial part of BA 5, which is related to a sensorimotor network (Leech et al., 2012; Vogt et al., 2006; Fink et al., 1997) (see Supplementary Figs. 3 and 4). Therefore, we confirm previous reports of abnormal interactions of CMS related to self-related processing with sensorimotor networks in schizophrenia (Ebisch and Aleman, 2016).

Regarding social perception, task-related fMRI results indicate impaired integrative processing of unimodal (visual) social information (others' facial expressions and tactile experiences). In contrast to the $\mathrm{HC}$ group, in the SCH group BOLD responses in dPCC were equal for stimuli where this information was congruent or incongruent and did not discriminate. Accordingly, PCC abnormalities have been related consistently to schizophrenia (Leech and Sharp, 2014).

Regarding our behavioral measures (see Supplementary material), we found delayed reaction times and increased chance performance in behavior when judging social stimulus congruence. These data are consistent with, and extend, previous studies in schizophrenia that demonstrate abnormal integrative processing of multisensory (audiovisual) emotional information (Muller et al., 2014; De Gelder et al., 2005) and congruence effects during the Stroop test (Westerhausen et al., 2011; Besnier et al., 2011).

Notably, we show that in the HC group increased congruence coding in APCC was predicted by decreased RS-FC with vMPFC. This implies that a greater independence between vMPFC and dPCC "resting state" activity allows dPCC activity to differentiate more dynamically between congruent and incongruent stimuli in the HC group. Indeed, these regions are related to distinct functional networks in healthy controls (Leech et al., 2012; Mantini et al., 2007).

In contrast to the HC group, vMPFC-dPCC RS-FC was altered in the $\mathrm{SCH}$ group, and no longer predicted stimulus-related activity during social perception in APCC. Thus, when "resting state" properties of self-related networks are abnormal, they may not be able to properly impact task-related activity anymore. Our data suggest that this could be linked to illness severity: negative symptoms were inversely related to the magnitude of differential task-evoked activity in dPCC for congruent and incongruent social stimuli. 
Some limitations of this study need to be mentioned. Firstly, sample size was relatively small. However, the 95\% CI visualized in Figs. 1 and 2 indicate narrow ranges of values and minimal overlap between the HC and $\mathrm{SCH}$ groups. This suggests accurate estimations and reliable differences in task and task-free neural activity patterns. Additionally, bootstrapping procedures indicated reliable estimates of correlation coefficients. Secondly, all patients were under pharmacological treatment. For ethical and clinical reasons, it was not possible to perform fMRI scanning in unmedicated patients. Nevertheless, only atypical antipsychotic drugs were used and covariance analyses showed that altered neural activity patterns in the SCH group were independent of antipsychotic medication dosage (CP values). Thirdly, whereas the $\mathrm{SCH}$ group showed impairment in both task-induced activity and behavioral performance during the social perception task, we could statistically relate only the former to RS-FC measures. Future studies are urged to clarify the relationship between RS-FC and behavioral measures.

In conclusion, we extend previous investigations on the link between self-disorder and social deficits in schizophrenia that mainly focused on impaired self-other distinction (van der Weiden et al., 2015; Ebisch et al., 2013; Nelson, Sass, et al., 2009). Specifically, the results show anomalies in RS-FC of self-related networks as well as in taskevoked neural activity (integrative processing) during social perception. Additionally, the relationship between this RS-FC and task-evoked activity is disrupted in the $\mathrm{SCH}$ group, too.

\section{Funding}

This work was supported by the following: Canadian Institute of Health Research (201103MOP-244752-BSB-CECA-179644), Michael Smith Foundation (200809EJL194083-EJL-CECA-179644), EJLB - Canadian Institute of Health Research (201103CCI248496-CCI-CECA-179644), CFI (Canadian Foundation for Innovation), National Natural Science Foundation of China (No. 31271195) and Canada Research Chairs to G Northoff; Wellcome Trust (grant no. 101253/A/13/Z) to D Mantini; Chiesi Foundation Parma, Italy, to V Gallese.

\section{Contribution}

Conception and design: SE, AS, GLR, MDG, VG, GN; data acquisition: SE, AS, MGP; participant recruitment: SE, AS, GM, GDI, MDG; data analysis: SE, DM, GN; data interpretation: SE, GN, VG, GM, MGP, GDI; drafting the manuscript: SE, GN, VG; revising the manuscript: AS, GM, GDI, DM, MGP, GLR, MDG. All authors contributed to and have approved the final manuscript.

\section{Conflict of interest}

The authors have declared that there are no conflicts of interest in relation to the subject of this study.

\section{Acknowledgments}

None.

\section{Appendix A. Supplementary data}

Supplementary data to this article can be found online at http://dx. doi.org/10.1016/j.schres.2017.07.020.

\section{References}

Addington, J., Penn, D., Woods, S.W., Addington, D., Perkins, D.O., 2008. Social functioning in individuals at clinical high risk for psychosis. Schizophr. Res. 99, 119-124.

Bai, Y., Nakao, T., Xu, J., Qin, P., Chaves, P., Heinzel, A., Duncan, N., Lane, T., Yen, N.S., Tsai, S.Y., Northoff, G., 2016. Resting state glutamate predicts elevated pre-stimulus alpha during self-relatedness: a combined EEG-MRS study on "rest-self overlap". Soc. Neurosci. 11, 249-263.

Berman, R.A., Gotts, S.J., McAdams, H.M., Greenstein, D., Lalonde, F., Clasen, L., Watsky, R.E., Shora, L., Ordonez, A.E., Raznahan, A., Martin, A., Gogtay, N., Rapoport, J., 2016. Disrupted sensorimotor and social-cognitive networks underlie symptoms in childhood-onset schizophrenia. Brain 139, 276-291.

Besnier, N., Kaladjian, A., Mazzola-Pomietto, P., Adida, M., Fakra, E., Jeanningros, R., Azorin, J.M., 2011. Differential responses to emotional interference in paranoid schizophrenia and bipolar mania. Psychopathology 44, 1-11.

Couture, S.M., Penn, D.L., Roberts, D.L., 2006. The functional significance of social cognition in schizophrenia: a review. Schizophr. Bull. 32 (Suppl. 1), S44-S63.

De Gelder, B., Vroomen, J., de Jong, S.J., Masthoff, E.D., Trompenaars, F.J., Hodiamont, P., 2005. Multisensory integration of emotional faces and voices in schizophrenics. Schizophr. Res. 72, 195-203.
Eack, S.M., Mermon, D.E., Montrose, D.M., Miewald, J., Gur, R.E., Gur, R.C., Sweeney, J.A Keshavan, M.S., 2010. Social cognition deficits among individuals at familial high risk for schizophrenia. Schizophr. Bull. 36, 1081-1088.

Ebisch, S.J., Aleman, A., 2016. The fragmented self: imbalance between intrinsic and extrinsic self-networks in psychotic disorders. Lancet Psychiatry 8, 784-790.

Ebisch, S.J., Gallese, V., Willems, R.M., Mantini, D., Groen, W.B., Romani, G.L., Buitelaar, J.K., Bekkering, H., 2011. Altered intrinsic functional connectivity of anterior and posterior insula regions in high-functioning participants with autism spectrum disorder. Hum. Brain Mapp. 32, 1013-1028.

Ebisch, S.J., Salone, A., Ferri, F., De Berardis, D., Romani, G.L., Ferro, F.M., Gallese, V., 2013. Out of touch with reality? Social perception in first-episode schizophrenia. Soc. Cogn. Affect. Neurosci. 8, 394-403.

Ebisch, S.J., Salone, A., Martinotti, G., Carlucci, L., Mantini, D., Perrucci, M.G., Saggino, A. Romani, G.L., Di Giannantonio, M., Northoff, G., Gallese, V., 2016. Integrative processing of touch and affect in social perception: an fMRI study. Front. Hum. Neurosci. 10, 209.

Fett, A.K., Viechtbauer, W., Dominguez, M.D., Penn, D.L., van Os, J., Krabbendam, L., 2011 The relationship between neurocognition and social cognition with functional outcomes in schizophrenia: a meta-analysis. Neurosci. Biobehav. Rev. 35, 573-588.

Fink, G.R., Frackowiak, R.S., Pietrzyk, U., Passingham, R.E., 1997. Multiple nonprimary motor areas in the human cortex. J. Neurophysiol. 77, 2164-2174.

Fisher, M., McCoy, K., Poole, J.H., Vinogradov, S., 2008. Self and other in schizophrenia: a cognitive neuroscience perspective. Am. J. Psychiatry 165, 1465-1472.

Fox, M.D., Raichle, M.E., 2007. Spontaneous fluctuations in brain activity observed with functional magnetic resonance imaging. Nat. Rev. Neurosci. 8, 700-711.

Fox, M.D., Zhang, D., Snyder, A.Z., Raichle, M.E., 2009. The global signal and observed anticorrelated resting state brain networks. J. Neurophysiol. 101, 3270-3283.

Gallese, V., 2003. The roots of empathy: the shared manifold hypothesis and the neural basis of intersubjectivity. Psychopathology 36, 171-180.

Gallese, V., 2007. Before and below 'theory of mind': embodied simulation and the neura correlates of social cognition. Philos. Trans. R. Soc. Lond. Ser. B Biol. Sci. 362, 659-669.

Green, M.F., Bearden, C.E., Cannon, T.D., Fiske, A.P., Hellemann, G.S., Horan, W.P., Kee, K., Kern, R.S., Lee, J., Sergi, M.J., Subotnik, K.L., Sugar, C.A., Ventura, J., Yee, C.M., Nuechterlein, K.H., 2012. Social cognition in schizophrenia, part 1: performance across phase of illness. Schizophr. Bull. 38, 854-864.

Haug, E., Oie, M., Andreassen, O.A., Bratlien, U., Raballo, A., Nelson, B., Moller, P., Melle, I. 2014. Anomalous self-experiences contribute independently to social dysfunction in the early phases of schizophrenia and psychotic bipolar disorder. Compr. Psychiatry 55, 475-482.

Horan, W.P., Green, M.F., DeGroot, M., Fiske, A., Hellemann, G., Kee, K., Kern, R.S., Lee, J Sergi, M.J., Subotnik, K.L., Sugar, C.A., Ventura, J., Nuechterlein, K.H., 2012. Social cognition in schizophrenia, part 2: 12-month stability and prediction of functional outcome in first-episode patients. Schizophr. Bull. 38, 865-872.

Huang, Z., Obara, N., Davis, H.H., Pokorny, J., Northoff, G., 2016. The temporal structure of resting-state brain activity in the medial prefrontal cortex predicts self-consciousness. Neuropsychologia 82, 161-170.

Kay, S.R., Fiszbein, A., Opler, L.A., 1987. The positive and negative syndrome scale (PANSS) for schizophrenia. Schizophr. Bull. 13, 261-276.

Kuhn, S., Gallinat, J., 2013. Resting-state brain activity in schizophrenia and major depression: a quantitative meta-analysis. Schizophr. Bull. 39, 358-365.

Leech, R., Braga, R., Sharp, D.J., 2012. Echoes of the brain within the posterior cingulate cortex. J. Neurosci. 32, 215-222.

Leech, R., Sharp, D.J., 2014. The role of the posterior cingulate cortex in cognition and disease. Brain 137, 12-32.

Mantini, D., Perrucci, M.G., Del Gratta, C., Romani, G.L., Corbetta, M., 2007. Electrophysiological signatures of resting state networks in the human brain. Proc. Natl. Acad. Sci. U. S. A. 104, 13170-13175

Martino, M., Magioncalda, P., Yu, H., Li, X., Wang, Q., Meng, Y., Deng, W., Li, Y., Li, M., Ma X., Lane, T., Duncan, N.W., Northoff, G., Li, T., 2017. Abnormal resting-state connectivity in a substantia nigra-related striato-thalamo-cortical network in a large sample of first-episode drug-Naïve patients with schizophrenia. Schizophr. Bull. http://dx.doi. org/10.1093/schbul/sbx067.

Minkowski, E., 1927. La schizophrenie. Psychopathologie des schizoides et des schizophrenes. Payot, Paris.

Mitchell, J.P., Banaji, M.R., Macrae, C.N., 2005. The link between social cognition and self-referential thought in the medial prefrontal cortex. J. Cogn. Neurosci. 17, $1306-1315$

Molnar-Szakacs, I., Uddin, L.Q., 2013. Self-processing and the default mode network: interactions with the mirror neuron system. Front. Hum. Neurosci. 7, 571.

Muller, V.I., Kellermann, T.S., Seligman, S.C., Turetsky, B.I., Eickhoff, S.B., 2014. Modulation of affective face processing deficits in schizophrenia by congruent emotional sounds. Soc. Cogn. Affect. Neurosci. 9, 436-444.

Nelson, B., Fornito, A., Harrison, B.J., Yucel, M., Sass, L.A., Yung, A.R., Thompson, A., Wood S.J., Pantelis, C., McGorry, P.D., 2009. A disturbed sense of self in the psychosis prodrome: linking phenomenology and neurobiology. Neurosci. Biobehav. Rev. 33, 807-817.

Nelson, B., Sass, L.A., Thompson, A., Yung, A.R., Francey, S.M., Amminger, G.P., McGorry, P.D., 2009. Does disturbance of self underlie social cognition deficits in schizophrenia and other psychotic disorders? Early Interv. Psychiatry 3, 83-93.

Northoff, G., 2014. How is our self altered in psychiatric disorders? A neurophenomenal approach to psychopathological symptoms. Psychopathology 47, 365-376.

Northoff, G., 2015. Resting state activity and the "stream of consciousness" in schizophrenia-neurophenomenal hypotheses. Schizophr. Bull. 41, 280-290.

Northoff, G., 2016. Is the self a higher-order or fundamental function of the brain? The "basis model of self-specificity" and its encoding by the brain's spontaneous activity. Cogn. Neurosci. 7, 203-222. 
Parnas, J., Bovet, P., Zahavi, D., 2002. Schizophrenic autism: clinical phenomenology and pathogenetic implications. World Psychiatry 1, 131-136.

Power, J.D., Barnes, K.A., Snyder, A.Z., Schlaggar, B.L., Petersen, S.E., 2012. Spurious but systematic correlations in functional connectivity MRI networks arise from subject motion. Neurolmage 59, 2142-2154.

Qin, P., Northoff, G., 2011. How is our self related to midline regions and the default-mode network? Neurolmage 57, 1221-1233.

Sass, L.A., Parnas, J., 2003. Schizophrenia, consciousness, and the self. Schizophr. Bull. 29 427-444.

Schilbach, L., Derntl, B., Aleman, A., Caspers, S., Clos, M., Diederen, K.M., Gruber, O., Kogler, L., Liemburg, E.J., Sommer, I.E., Muller, V.I., Cieslik, E.C., Eickhoff, S.B., 2016. Differentia patterns of dysconnectivity in mirror neuron and mentalizing networks in schizophrenia. Schizophr. Bull. 42, 1135-1148.

Schilbach, L., Eickhoff, S.B., Rotarska-Jagiela, A., Fink, G.R., Vogeley, K., 2008. Minds at rest? Social cognition as the default mode of cognizing and its putative relationship to the "default system" of the brain. Conscious. Cogn. 17, 457-467.

Sheffield, J.M., Barch, D.M., 2016. Cognition and resting-state functional connectivity in schizophrenia. Neurosci. Biobehav. Rev. 61, 108-120.

Sui, J., Humphreys, G.W., 2015. The integrative self: how self-reference integrates perception and memory. Trends Cogn. Sci. 19, 719-728.

van der Meer, L., Costafreda, S., Aleman, A., David, A.S., 2010. Self-reflection and the brain: a theoretical review and meta-analysis of neuroimaging studies with implications for schizophrenia. Neurosci. Biobehav. Rev. 34, 935-946. van der Weiden, A., Prikken, M., van Haren, N.E., 2015. Self-other integration and distinction in schizophrenia: a theoretical analysis and a review of the evidence. Neurosci. Biobehav. Rev. 57, 220-237.

Van Dijk, K.R., Hedden, T., Venkataraman, A., Evans, K.C., Lazar, S.W., Buckner, R.L., 2010. Intrinsic functional connectivity as a tool for human connectomics: theory, properties, and optimization. J. Neurophysiol. 103, 297-321.

Vinogradov, S., Luks, T.L., Schulman, B.J., Simpson, G.V., 2008. Deficit in a neural correlate of reality monitoring in schizophrenia patients. Cereb. Cortex 18, 2532-2539.

Vogt, B.A., Vogt, L., Laureys, S., 2006. Cytology and functionally correlated circuits of human posterior cingulate areas. NeuroImage 29, 452-466.

Weinberger, D.R., Radulescu, E., 2016. Finding the elusive psychiatric "lesion" with 21stcentury neuroanatomy: a note of caution. Am. J. Psychiatry 173, 27-33.

Westerhausen, R., Kompus, K., Hugdahl, K., 2011. Impaired cognitive inhibition in schizophrenia: a meta-analysis of the Stroop interference effect. Schizophr. Res. 133, $172-181$.

Whitfield-Gabrieli, S., Moran, J.M., Nieto-Castanon, A., Triantafyllou, C., Saxe, R., Gabrieli, J.D., 2011. Associations and dissociations between default and self-reference networks in the human brain. Neurolmage 55, 225-232.

Yang, G.J., Murray, J.D., Repovs, G., Cole, M.W., Savic, A., Glasser, M.F., Pittenger, C., Krystal, J.H., Wang, X.J., Pearlson, G.D., Glahn, D.C., Anticevic, A., 2014. Altered global brain signal in schizophrenia. Proc. Natl. Acad. Sci. U. S. A. 111, 7438-7443. 